\title{
Stability and Activation Gaps of the Parafermionic Hall States in the Second Landau Level
}

\author{
L. S. Georgiev ${ }^{1}$ \\ Institut für Theoretische Physik, Universität zu Köln, Zülpicher Str. 77, 50937 \\ Köln, GERMANY
}

\begin{abstract}
Analyzing the effective conformal field theory for the parafermionic Hall states, corresponding to filling fractions $\nu_{k}=2+k /(k M+2), k=2,3, \ldots, M$ odd, we show that the even $k$ plateaux are expected to be more stable than their odd $k$ neighbors. The reason is that the parafermion chiral algebra can be locally extended for $k$ even. This reconciles the theoretical implication, that the bigger the $k$ the less stable the fluid, with the experimental fact that, for $M=1$, the $k=2$ and $k=4$ plateaux are already observed at electron temperature $T_{e} \simeq 8 \mathrm{mK}$, while the Hall resistance for $k=3$ is not precisely quantized at that temperature in the sample of Pan et al. Using a heuristic gap ansatz we estimate the activation energy gap for $\nu_{3}=13 / 5$ to be approximately $0.015 \mathrm{~K}$, which implies that the quantization of the Hall conductance could be observed for temperature below $1 \mathrm{mK}$ in the same sample. We also find an appealing exact relation between the fractional electric charge and fractional statistics of the quasiholes. Finally, we argue that besides the Moore-Read phase for the $\nu_{2}=5 / 2$ state there is another relevant phase, in which the fundamental quasiholes obey abelian statistics and carry half-integer electric charge.
\end{abstract}

Key words: Quantum Hall effect, Conformal field theory, Parafermions PACS: 11.25.H, 71.10.Pm, 73.43.Cd

Email address: lg@thp.uni-koeln.de (L. S. Georgiev).

1 On leave of absence from Institute for Nuclear Research and Nuclear Energy, Tsarigradsko Chaussee 72, BG-1784 Sofia, BULGARIA 


\section{Introduction}

In 1998 Read and Rezayi have found [1] a new hierarchy of incompressible ground states, in the second Landau level, for quantum Hall (QH) fluids corresponding to filling fractions

$$
\begin{array}{ll}
\nu_{k}=2+\frac{k}{k M+2}, & k=2,3,4, \ldots \\
M \text { odd },
\end{array}
$$

(the number 2 in Eq. (1) comes from the two completely filled lowest Landau levels with electrons of both spins). For what follows, it will be convenient to introduce also the particle-hole $(\mathrm{PH})$ conjugated $\mathrm{QH}$ fluids corresponding to filling fractions

$$
\nu_{k}^{\prime}=2+\left(1-\frac{k}{k M+2}\right)
$$

where the PH conjugation concerns only the last occupied Landau level (assumed polarized). We shall concentrate on the case $M=1$, though the general $M$ will be kept in most of the formulae. The first plateau $(k=2)$ in the series (1) and (2) is the mysterious $[2,3] \nu=5 / 2$, whose nature is still unclear and whose ground state wave function is believed to have a good overlap with that of the Pfaffian state [4-8]. The ground state wave functions for the $\mathrm{QH}$ fluids with filling factors (1) have been explicitly constructed in [1] by using the operator product expansion (OPE) of $\mathbb{Z}_{k}$ parafermionic currents [9-11] describing the low-energy effective field theory for the edge excitations. A very important feature of these wave functions is their cluster decomposition property [1,12-14], implying that the electrons form clusters of $k$-particles which then interact almost like in a Laughlin fluid.

Shortly after the work [1] Pan et al. [15] have performed a very precise measurement of the activation energy gap for $\nu_{2}=5 / 2$, at very low electron temperature, in an extremely high-mobility sample and, for the first time, have found that this plateau is quantized to within $2 \mathrm{ppm}$. They have also measured the energy gaps corresponding to the strong minima of $R_{x x} \simeq 0$ at $\nu_{4}=8 / 3$ and $\nu_{4}^{\prime}=7 / 3$. In addition, well developed minima at $\nu_{3}=13 / 5$ and $\nu_{5}=19 / 7$ (with $\mathrm{PH}$ conjugates $\nu_{3}^{\prime}=12 / 5$ and $\nu_{5}^{\prime}=16 / 7$, respectively) have been seen, however, gap measurements for these plateaux have not been reported since $R_{x x} \neq 0$. Thus, the first plateaux $(k=2,3,4)$ in the sequences (1) and (2) for $M=1$ have been experimentally observed [15]. A striking fact is that according to the experiment [15] the $k=2$ and $k=4$ plateaux are

clearly quantized while the $k=3$ one is not. On the other hand, the theoretical 
investigation of the stability of the quantum Hall liquids [16] (see the stability criteria S1 and S3 in Sect. 3.3 there) implies for the hierarchies (1) and (2) that the bigger the $k$ the less stable the fluid, i.e., one should expect that the $k=3$ state is more stable (respectively, has a bigger activation gap) than the $k=4$ one and the former should be "more visible" than the latter at the same temperature. Here we speak about stability with respect to compression and decompression of the electron fluid, i.e., with respect to deviation of the magnetic field from the center of the plateau. In other words, stability could be expressed in terms of the energy gap of the $\mathrm{QH}$ fluid.

In this paper we are trying to give an explanation of the above contradiction between theory and experiment. More precisely, using the conformal field theory (CFT) [17] description of the edge states [18,19], we will show that the even $k$ plateaux are more stable than their odd $k$ neighbors, i.e., the energy gaps for the former are expected to be bigger than those for the latter. To this end, we make use of recent studies $[13,14]$ of the parafermionic Hall states in terms of a CFT coset construction for the corresponding edge states and the stability criteria in [16]. In particular, we use the chiral partition functions (or CFT characters for all possible boundary conditions which characterize the universal properties of the edge excitations), derived in [13,14], to identify the quasiholes of the corresponding fluids and find their CFT dimensions, charges, fluxes and fusion rules (or rules for making composite particles).

We have to make some terminology remarks here. We use the CFT as a lowenergy (or large-scale) effective field theory describing the spectrum (charge, spin and statistics) of the edge excitations $[20,18,19,16]$. There is a selected class of edge excitations which are characterized by the fact that their manybody wave functions, which are realized as CFT correlators, are single-valued in the (complex plane) coordinates of all particles. In the algebraic approach these excitations are represented by relatively local $[20,19,16]$ quantum fields which contain only integer negative powers of the distance in their singular short-distance OPEs and generically form an operator algebra (see, e.g., the condition $\mathrm{C} 4$ in [16]). This algebra completely characterizes the universality class of the QH fluid and is called the chiral (observable) algebra [16], because the excitations on the edge are chiral; all the excitations (charged and neutral) in it can be generated from the vacuum by applying only local chiral operators. As a consequence, all charged excitations in that algebra carry integer electric charge (in units e $=1$ ) and satisfy the standard charge-statistics relation [20,19] for Bose/Fermi particles in the QH effect context; in particular, the relative locality with themselves imply that the CFT dimensions of these fields must be integer/half-integer. The chiral algebra always contains the Virasoro stress energy tensor [17] whose commutation relations are characterized by a (positive) number $c$, called the central charge, which is related to the Casimir energy on the cylinder [17]. On the other hand, there are excitations, such as the Laughlin quasihole, whose wave functions are single-valued 
in the coordinates of the electrons (or of the chiral observables in general) but are multi-valued in their own positions 2 . Such excitations cannot be obtained directly from the vacuum (by acting with local operators) but are instead generated by non-local "intertwiners" [17]. The latter fields (called also non-local primary fields) are relatively local with the chiral algebra but are not local with themselves; as a consequence, these fields can have fractional charges and obey fractional statistics [21]. From the algebraic point of view, such intertwiners form representations [16] of the chiral algebra and those of them which have electric charge less than 1 (in absolute value) give the inequivalent irreducible representations (IR)s $[19,16]$. The number of the inequivalent IRs was called the topological order [22]. Finally, the CFT describing the edge excitations of the QH fluid is expected to be well-defined on the torus and is therefore supposed to be $S L(2, \mathbb{C})$ covariant [16], i.e., it should be a rational CFT 3 . In other words, there is a one-to-one correspondence between the CFTs and $\mathrm{QH}$ universality classes. Thus, it is always crucial to have a complete list of IRs of the QH chiral algebra since they characterize the topological properties of all possible quasiparticles in the $\mathrm{QH}$ fluid.

Next, a few words about stability, justifying the use of CFT cosets for the QH effect, are in order. As a first step, one would like to reduce (or gauge away) as much as possible the neutral chiral subalgebra (respectively the neutral quasiholes' state space) for a QH edge since this generically decreases the Virasoro central charge (without changing the QH filling factor) and, according to the stability criterion S1 in [16], makes the QH fluid more stable. In our case $[13,14]$, the coset central charge

$$
c_{k}^{\mathrm{PF}}=\frac{2(k-1)}{k+2}
$$

is smaller than the (neutral part of the) original one $c_{k}^{(0)}=2(k-1)$. On the other hand, however, reducing the algebra in principle increases the number of IRs and, therefore, decreases the fluid's stability (S3 criterion in [16]). However, after any reduction of the chiral algebra, some of the new IRs may become equivalent, hence the number of inequivalent ones actually decreases. This is exactly what happens in the coset - due to the very "symmetric" reduction, there are a lot of equivalences, known as field identifications under the action of the simple currents [24] (see also Appendix B in [16]), which effectively

$\overline{2}$ Usually the Laughlin quasihole wave functions are written without the factors containing the relative coordinates of the quasiholes. In the CFT approach these pieces appear automatically when computing the conformal blocks corresponding to the wave functions.

3 there is a selected class of theories, known as $W_{1+\infty}$ minimal models [23], which are not rational CFTs but may succesfully describe the incompressible QH fluids in the principle series 
reduce the number of IRs and therefore the coset fluid [16,14] appears to be much more stable than the original multi-component Luttinger liquid [16]. This first step for the parafermion QH fluids has been accomplished in [14]. As a second step towards a bigger stability, one looks for the maximal (local) extension of the chiral algebra at fixed central charge, since this naturally decreases the number of non-equivalent IRs and further increase the stability of the QH fluid, according to the stability criterion S3 in [16]. However, only bosonic and fermionic fields can be used for such an extension because of the locality requirement [16].

Our main result is based on the fact, already observed in [1], that for $k=$ $2 \kappa$, there are composite fermions/bosons (for $\kappa$ odd/even) among the $\mathbb{Z}_{k}$ parafermions which are relatively local and therefore can be added to the chiral algebra. As a consequence, this changes the quasihole identification and increases their CFT dimensions. We find that the quasihole's CFT dimensions are $($ for $M=1)$

$$
\Delta_{k}^{\text {q.h. }}=\left\{\begin{array}{c}
\frac{1}{k+2} \text { for } k \text { even } \\
\frac{1}{2(k+2)} \text { for } k \text { odd }
\end{array}\right.
$$

i.e., the quasiholes for the even plateaux have twice bigger dimensions. A similar relation is found as well for the electric charges of the quasiholes. On the other hand, Park and Jain [25] have found that the energy gaps for the principle series of $\mathrm{QH}$ plateaux have universal components proportional to the quasiparticle's electric charge. We conjecture that the energy gap for the parafermion $Q H$ fluid has a universal component too, which should be expressed in terms of the universal characteristics of the quasiparticles, such as the electric charge and the quantum statistics; we find that, for the elementary quasiholes in the parafermion $\mathrm{QH}$ states the charge is proportional to their CFT dimension (or statistics) (see the discussion in Sect. 4). Therefore, we could use Eq. (4) to compute the activation energy gaps. Taking into account the gap reduction $\Gamma$ and the renormalization $\alpha$ due to the non-zero thickness, Landau level mixing and residual disorder [25] we use the following measurable gap ansatz (cf. Eq. (3) in [25])

$$
\widetilde{\Delta}_{k}=\alpha \Delta_{k}^{\text {q.h. }} \frac{e^{2}}{4 \pi \varepsilon l_{k}}-\Gamma
$$

where $\Delta_{k}^{\text {q.h. }}$ is given by Eq. (4), $l_{k}=\sqrt{\hbar / e B_{k}}$ is the magnetic length, $\varepsilon$ is the dielectric constant $\left(\varepsilon \simeq 12.6 \varepsilon_{0}\right.$ for GaAs) and $B_{k}$ is the magnetic field at the center of the plateau corresponding to the filling factor $\nu_{k}$ (see Table 1 ). 
Note that Eq. (5) is not a result of exact or numerical diagonalization of a particular electron interaction. It is based on the conjecture that the gap has a universal component (like in the principle series [25] where this was found by numerical calculation) and on the stability criteria in [16].

Fitting the gaps data from [15] for the two most stable plateaux in the hierarchy (1), $\widetilde{\Delta}_{2}=0.11 \mathrm{~K}$ and $\widetilde{\Delta}_{4}=0.055 \mathrm{~K}$, we find that $\alpha=0.0063$ and $\Gamma=0.045 \mathrm{~K}$ for the sample of [15]. This allows us to estimate the energy gaps for the plateaux in the hierarchies (1) and (2) (see Tables 1 and 2 respectively). The gap for the $\nu=13 / 5 \mathrm{QH}$ state is estimated to be $\sim 15 \mathrm{mK}$, which explains the absence of a Hall plateau in the experiment [15]. This also suggests that the quantization is to be observed for $T_{\mathrm{e}}<1 \mathrm{mK}$. Next, we expect that the less expressed $R_{x x}$ minimum at magnetic field $B \simeq 3.35 \mathrm{~T}$ in the same sample corresponds to the plateau $11 / 4(k=6)$, whose estimated activation gap is $0.029 \mathrm{~K}$ (plus some interference from the $14 / 5(k=8)$ state), rather than to $19 / 7(k=5)$ as supposed by Pan et al. The plateaux with odd $k>5$ are not expected to be observed in this sample even at zero temperature and the last observable even $k$ plateau in this series is expected to be $14 / 5$.

In addition, a very interesting property of the parafermion $\mathrm{QH}$ fluid is found: the quasiparticle excitations satisfy a generalized charge-statistics relation which explicitly connects the fractional electric charge to the fractional quantum statistics. In fact, we believe that this relation is a fundamental characteristics of $\mathrm{QH}$ quasiparticles.

Finally, according to our analysis of the stability of the parafermion QH states, we conclude that, at higher temperature, the $k=2$ state may prefer quasiparticles with fractional charge $1 / 2$, which obey abelian statistics with $\theta=\pi / 2$. This defines a new universality class relevant for the $\nu=5 / 2 \mathrm{QH}$ state, which is different from that of the Moore-Read (MR) state and a (finite temperature) transition between the two phases is possible (see the discussion in Sect. 5).

The rest of this paper is organized as follows: in Sect. 1 we review basic facts about the realization of parafermions as a coset CFT, in Sect. 2 we discuss the consequences of the extension of the chiral algebra in terms of the composite fermions/bosons, in Sect. 3 we investigate the relation between charge and statistics of QH quasiparticles and in Sect. 4 we compute the energy gaps for the parafermionic states using the gap ansatz (5). The numerical results are summarized in Tables 1 and 2 . 


\section{The parafermions as a coset}

The parafermions are realized in $[13,14]$ (see also the references therein) as a "diagonal" affine coset [26] made in an abelian CFT, which describes the edge of a multi-component chiral Luttinger liquid. More precisely, the topological charge lattice (whose metrics is also known as the $K$-matrix) is of the type $\left(M+\left.2\right|^{1} A_{k-1}{ }^{1} A_{k-1}\right)$ in the notation of [20], where it was called a maximally symmetric (chiral) quantum Hall lattice. The charge vector $\mathrm{Q}$, whose square gives the filling factor [20], in this $K$-matrix model has components $\underline{\mathrm{Q}}=$ $(1,0, \ldots, 0)$ in the dual lattice $[20,13,14]$ implying that this liquid possesses a large number of neutral degrees of freedom, which could be described by the Kac-Moody algebra $[20,13,14] \widehat{s u(k)_{1}} \oplus \widehat{s u(k)_{1}}$. The $\mathbb{Z}_{k}$-parafermions [11] are obtained here by the affine coset $[26,14]$

$$
\mathrm{PF}_{k}=\frac{\widehat{s u(k)_{1}} \oplus \widehat{s u(k)_{1}}}{\widehat{s u(k)_{2}}},
$$

where the "diagonal" subalgebra $\widehat{s u(k)_{2}}$ in the denominator is generated by $J^{a}=J_{(1)}^{a}+J_{(2)}^{a}$ and $J_{(i)}^{a}(z)$ are the currents in the two copies of $\widehat{s u(k)_{1}}$ in the numerator $[13,14]$. The central charge for the coset (6) is given by $[13,14]$ Eq. (3). The above mentioned $s u(k) \times s u(k)$ symmetry becomes more transparent if we write the dual pair isomorphism [17]

$$
\widehat{u(1)} \oplus \widehat{s u(k)_{1}} \oplus \widehat{s u(k)_{1}} \simeq \widehat{s u(2 k)_{1}} \simeq \widehat{s u(2)_{k}} \oplus \widehat{s u(k)_{2}}
$$

The $\widehat{s u(2)_{k}}$ Kac-Moody algebra here is simply the usual spin algebra of $k$ pairs of (chiral, complex) Dirac-Weyl fermions corresponding to $k$ different layers (one pair for each layer), while the $\widehat{s u(k)_{2}}$ is just their layer symmetry. Gauging away the latter subalgebra actually removes the layer symmetry, i.e., projects all particles to the same layer, which gives another viewpoint for the understanding of the clustering mechanism and the non-abelian statistics of quasiparticles in these fluids [14].

We have to stress that the consistency of the neutral projection in Eq. (6) requires decoupling of neutral and charged degrees of freedom[14]. However, in the original $K$-matrix theory they are not completely separated $[13,14]$. For the solution of this problem the spin-charge separation is obtained in a decomposable sub-lattice of the original one $[13,14]$. The price one has to pay is the appearance of a $\mathbb{Z}_{k}$ pairing rule (PR) [13,14] for combining neutral and charged excitations of the decomposable lattice. More precisely, the IRs of the original $K$-matrix chiral algebra can be labeled [14] by the (fractional 
part of the) $\widehat{u(1)}$ charge $l$ and the $\widehat{s u(k)_{1}} \oplus \widehat{s u(k)_{1}}$ weights $\left(\underline{\Lambda}_{\mu}, \underline{\Lambda}_{\rho}\right)$, where $\underline{\Lambda}_{\mu}, \mu=0, \ldots, k-1$ are the $\operatorname{su}(k)$ fundamental weights $\left(\underline{\Lambda}_{0}=0\right)$, such that the PR holds, i.e., $\mu+\rho=l \bmod k$. Furthermore, the coset reduction in the neutral part is generically supposed to preserve this $\mathrm{PR}$ - now it couples the parafermionic $\mathbb{Z}_{k}$-charge to the electric charge [14]. This inheritance of excitation pairing is one of the main advantages of the coset realization (6) of the $\mathbb{Z}_{k}$ parafermions as compared to the more standard $\widehat{s u(2)_{k}} / \widehat{u(1)}$ coset [27]. Another advantage of the diagonal coset approach [14] is that the original model is abelian, i.e., better understood, in which some more complicated nonabelian objects are projected out.

The IRs for the coset (6), which describe the neutral excitations of the parafermionic QH fluid, are labeled by sums of two $s u(k)$ fundamental weights $[13,14]$

$$
\underline{\Lambda}_{\mu}+\underline{\Lambda}_{\rho}, \quad 0 \leq \mu \leq \rho \leq k-1
$$

(these actually represent all admissible $\widehat{s u(k)_{2}}$ weights [17]). The conformal dimensions of the corresponding coset primary fields $\Phi^{\mathrm{PF}}\left(\underline{\Lambda}_{\mu}+\underline{\Lambda}_{\rho}\right)$ are given by $[14]$

$$
\Delta^{\mathrm{PF}}\left(\underline{\Lambda}_{\mu}+\underline{\Lambda}_{\rho}\right)=\frac{\mu(k-\rho)}{k}+\frac{(\rho-\mu)(k-(\rho-\mu))}{2 k(k+2)}, \quad 0 \leq \mu \leq \rho \leq k-1 .(7)
$$

As mentioned before, the parafermion fluid inherits from the parent $K$-matrix model a $\mathbb{Z}_{k}$ PR, which can now be written as follows $[13,14]$

$$
P=l \bmod k, \quad \text { where } \quad P=P\left(\underline{\Lambda}_{\mu}+\underline{\Lambda}_{\rho}\right)=\mu+\rho \bmod k
$$

and $l$ is the electric charge label introduced above. This approach allows us to compute all characteristics of the parafermionic fluid using those of the Luttinger liquid plus the coset reduction. In particular, the chiral (grand canonical) partition functions for the complete parafermionic theory ( $l$ and $\rho$ label the electric and neutral sectors respectively [14])

$$
\chi_{l, \rho}(\tau, \zeta)=\operatorname{tr}_{\mathcal{H}_{l, \rho}} q^{L_{0}-c / 24} \mathrm{e}^{2 \pi i \zeta J_{0}^{\mathrm{el}}}
$$

where $L_{0}-c / 24$ is the chiral Hamiltonian and $J_{0}^{\text {el }}$ is the zero mode of the electric current $J^{\mathrm{el}}(z)$ (normalized as usual by $\left.J^{\mathrm{el}}(z) J^{\mathrm{el}}(w) \sim \nu_{k} /(z-w)^{2}\right)$ could be written as follows $[13,14]$

$$
\chi_{l, \rho}(\tau, \zeta)=\sum_{s=0}^{k-1} K_{l+s(k M+2)}(\tau, k \zeta ; k(k M+2)) \operatorname{ch}\left(\underline{\Lambda}_{l-\rho+s}+\underline{\Lambda}_{s+\rho}\right),
$$


where $l$ and $\rho$ are respectively $\bmod (k M+2)$ and $\bmod k$ indices. Here $K_{\lambda}(\tau, k \zeta ; k(k M+2))$ are the $\widehat{u(1)}$ (rational torus of compactification radius $\sqrt{k(k M+2)})$ chiral partition functions (here $q=\mathrm{e}^{2 \pi i \tau}, 2 \pi R \operatorname{Im} \tau=1 / k_{B} T$, $2 \pi \operatorname{Re} \zeta=V_{0} / k_{B} T, 2 \pi \operatorname{Im} \zeta=\mu / k_{B} T$, where $T$ is the temperature, $k_{B}-$ the Boltzmann constant, $\mu$ - the chemical potential and $V_{0}$ is the Hall difference between the edges [28]), and $\operatorname{ch}\left(\underline{\Lambda}_{\mu}+\underline{\Lambda}_{\rho}\right)$ are the parafermion (neutral) partition functions written in the universal chiral partition function (UCPF) form $[13,14,29] \operatorname{ch}\left(\underline{\Lambda}_{\mu}+\underline{\Lambda}_{\rho}\right) \equiv \operatorname{ch}_{\rho-\mu}^{\rho}\left(\tau ; \mathrm{PF}_{k}\right)$, where $0 \leq \mu \leq \rho \leq k-1$. The concrete forms of these partition functions can be found in $[13,14]$, however, they are not important for our discussion and we only mention that all the sectors could be obtained uniquely [14] if $0 \leq \rho-\mu \leq \rho \leq k-1$.

Each term in the sum in Eq. (9) satisfies the PR (8) and represents some (basic) quasiparticle excitation

$$
\Psi_{l, \rho, s}(z)=: \mathrm{e}^{i \frac{l+s(k M+2)}{\sqrt{k(k M+2)} \phi(z)}}: \Phi^{\mathrm{PF}}\left(\underline{\Lambda}_{l-\rho+s}+\underline{\Lambda}_{\rho+s}\right)(z)
$$

of the parafermionic QH fluid. The electric charge $Q$ and the total conformal dimension $\Delta$ of (10) read [14]

$$
\begin{aligned}
& Q=k \frac{l+s(k M+2)}{k(k M+2)}=s+\frac{l}{k M+2}, \\
& \Delta=\frac{(l+s(k M+2))^{2}}{2 k(k M+2)}+\Delta^{\mathrm{PF}}\left(\underline{\Lambda}_{l-\rho+s}+\underline{\Lambda}_{s+\rho}\right),
\end{aligned}
$$

where $\Delta^{\mathrm{PF}}$ is given by Eq. (7) and $l$ and $\rho$ take the same values as in Eq. (9). The physical hole operator $(l=\rho=0, s=1)$ is represented by the tensor product satisfying the PR (8) [14]

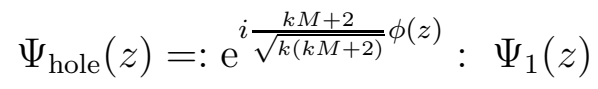

of the parafermion current $\Psi_{1}=\Phi^{\mathrm{PF}}\left(\underline{\Lambda}_{1}+\underline{\Lambda}_{1}\right)$ with the $\widehat{u(1)}$ part vertex exponent $\left(\phi(z)\right.$ being the chiral boson [17] such that $\left.i \partial \phi(z)=J^{\mathrm{el}}(z) / \sqrt{\nu_{k}}\right)$. It is characterized by the $s=1$ term in $\chi_{0,0}$ and has the following charge, flux (in units of flux quanta) and conformal dimension

$$
Q_{\text {hole }}=1, \quad \Phi_{\text {hole }}=\frac{k M+2}{k}, \quad \Delta_{\text {hole }}=\frac{M+2}{2} .
$$

Notice that the dimension of the hole (respectively, of the electron) is independent of $k$ and for $M=1$ is always $3 / 2$ so that the stability criterion (S2) in [16] gives no restriction here. 
Remark 1. The fact that the flux (13) attached to holes (respectively, to electrons) is not integer in the vacuum super-selection sector, implies that these could not live alone, because of flux quantization, but should rather form clusters of $k$ objects [1,12-14] (the only exception is the case $k=2$ where the hole's flux is integer and the edge electrons are not paired [5]). We stress that such a phenomenon of electron clustering is always supposed to take place when the numerator of the filling factor is bigger than 1, e.g., in the principle series of filling fractions.

Similarly, the quasihole operator $(l=1, \rho=s=0)$ is identified with [14]

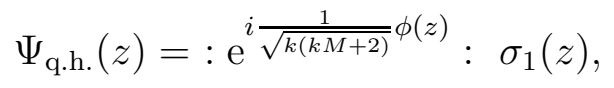

where $\sigma_{1}=\Phi^{\mathrm{PF}}\left(\underline{\Lambda}_{0}+\underline{\Lambda}_{1}\right)$ is the "spin" field corresponding to the lowest charge and dimension IR of the coset. It is also worth-noting that it generates all the other IRs by multiple fusion with itself. The field (14) is characterized by the $s=0$ term in $\chi_{1,0}$ so that its charge and total conformal dimension are

$$
Q_{\text {q.h. }}=\frac{1}{k M+2}, \quad \Phi_{\text {q.h. }}=\frac{1}{k}, \quad \Delta_{\text {q.h. }}^{(M=1)}=\frac{1}{2(k+2)}
$$

Again, the quasiholes are enforced to form clusters of $k$ because of flux quantization.

The electric charge of the quasihole coincides with the minimal possible charge $Q_{\min }=1 /\left(\ell d_{H}\right)$ in a $\mathrm{QH}$ fluid, according to Eqs. (67-69) in [16], where $d_{H}=$ $k M+2$ is the denominator of the filling factor and $\ell=1$ is the charge parameter $[16,20,19]$. To make contact with the notation in [16] we notice that the the conformal dimension of the parafermion $\Psi_{1}$ is $(k-1) / k$ so that the order [16] of this simple current is ord $\left(\Psi_{1}\right)=k$, while $r\left(\Psi_{1}\right)=2$ coincides with its $\mathbb{Z}_{k}$-parafermion charge.

\section{Composite particles and the maximal chiral algebra extension for $k=2 \kappa$}

As already mentioned in the Introduction, the case $k=2 \kappa$, with $\kappa$ a positive integer, is more delicate. The reason is that, according to Eq. (7), the parafermion primary field labeled by [14] $\underline{\Lambda}_{\kappa}+\underline{\Lambda}_{\kappa}$ (which is, in fact, the parafermion current denoted by $\psi_{\kappa}$ in [9]) in the neutral sector

$$
\Psi_{\kappa}^{(0)}=\Phi^{\mathrm{PF}}\left(\underline{\Lambda}_{\kappa}+\underline{\Lambda}_{\kappa}\right), \quad \Delta^{\mathrm{PF}}\left(\underline{\Lambda}_{\kappa}+\underline{\Lambda}_{\kappa}\right)=\kappa / 2
$$


has a half-integer/integer (neutral) conformal dimension $\Delta^{\mathrm{PF}}$ for $\kappa$ odd/even. Next, in order to form an allowed quasiparticle excitation, this parafermion current must be combined with some charged operator : $\mathrm{e}^{i \frac{l}{\sqrt{k(k M+2)}} \phi(z)}$ : in such a way that the PR (8) holds, i.e., $2 \kappa=l \bmod k$, which has only one solution $4, l=0$, in the interval $-(k M+2) / 2 \leq l \leq(k M+2) / 2-1$ (i.e., $l \bmod (k M+2)$ like in Eq. (9) ) for $M=1$ and $k>2$. Therefore, $l=0$ defines a unique neutral chiral "observable" composite particle

$$
\Psi_{\kappa}(z)=1 \otimes \Psi_{\kappa}^{(0)}(z), \quad \Delta_{\kappa}=\Delta_{\kappa}^{\mathrm{PF}}=\kappa / 2, \quad Q_{\kappa}=\Phi_{\kappa}=0,
$$

which could be physically interpreted [1] as made from $\kappa$ particles $\Psi_{\mathrm{p}}=$ : $\mathrm{e}^{-i \frac{1}{\sqrt{\nu}} \phi(z)}: \Phi^{\mathrm{PF}}\left(\underline{\Lambda}_{k-1}+\underline{\Lambda}_{k-1}\right)$ and $(k M+2) / 2$ flux $\square: \mathrm{e}^{i \frac{k}{\sqrt{k(k M+2)}} \phi(z)}:$. These neutral composites have half-integer/integer total conformal dimensions $\Delta_{\kappa}$, are always (super)local with themselves (in addition to being local with the entire chiral algebra - see the relative locality discussion in the Introduction) and by convention should belong to the chiral (super)algebra rather than to the set of its representations. In other words, the original chiral algebra must be extended by the neutral composite (17) since it is simply a boson for $\kappa$ even and a fermion for $\kappa$ odd according to the standard spin-statistics relation $2 \Delta_{\kappa}=\theta / \pi \bmod 2(\Delta$ in this case gives the conformal spin). Note also that the existence of the field (17) does not contradict the charge-statistics relation (C3 in [16]) since this field is neutral. Moreover, such a field must exist in order to restore the standard charge-statistics relation for the cluster containing $\kappa$ electrons. The point is that the flux composite containing $\kappa+1$ flux corresponds to electric charge $\kappa$, however, it is always a boson since its conformal dimension is $\kappa(\kappa+1) / 2$, which is integer. Therefore, to create a cluster of $\kappa$ electrons one needs a neutral particle, such as (17), which is a boson/fermion when $\kappa$ is even/odd.

The above extension defines a different rational CFT, which satisfies all necessary conditions [16] for a QH edge effective field theory. Therefore, the extended rational CFT identifies a new universality class for the $\nu=5 / 2$, which is different from that for the MR state.

The neutral composites (17) are fundamental in the sense that their correlation functions are single-valued and, as a consequence of Ward identities, the parafermionic component of the stress tensor could be expressed as

$$
T^{\mathrm{PF}}(w)=\frac{k-1}{k(k+2)} \lim _{z \rightarrow w}\left(\frac{\partial}{\partial z}-\frac{\partial}{\partial w}\right)^{2}\left[(z-w)^{\kappa} \Psi_{\kappa}(z) \Psi_{\kappa}(w)\right] .
$$

4 only for $k=2, M=1$ the solutions are two: $l=-2,0$

5 Note that the field representing a quantum of flux does satisfy the PR (8) (i.e., $0=k \bmod k)$ and is therefore a valid excitation of the parafermion $\mathrm{QH}$ fluid 
The importance of the neutral composite (17) stems from the fact that the ordinary electron dropped into the $\mathrm{QH}$ fluid, for $k=2 \kappa$, splits into $(k M+$ 2)/2 elementary quasiparticles of both kind (q.p.1 and q.p.2 in the lattice description, i.e., before making the coset projection $[13,14])$ and one composite (17)

$$
1 \mathrm{e} \simeq \frac{k M+2}{2}(\text { q.p. } 1+\text { q.p. } 2)+1 \text { comp. }
$$

To demonstrate that, we note that the pair (1 hole -1 electron) has no long-range Aharonov-Bohm effects [30] (i.e., it is characterized by a trivial topological charge) and therefore must be invisible in the adiabatic transport of any excitation around it. Consider a cluster consisting of 1 hole, described [14] by the lattice charge $\underline{\mathrm{q}}_{\mathrm{hole}}=(k M+2) \underline{\mathrm{e}}_{1}^{*}+\underline{\Lambda}_{1}^{(\alpha)}+\underline{\Lambda}_{1}^{(\beta)}$, and $(k M+2) / 2$ quasiparticles of both kinds, described by the lattice charges [14] $\underline{\mathrm{q}}_{\mathrm{q} . \mathrm{p} .1}=$ $-\underline{\mathrm{e}}_{1}^{*}+\underline{\Lambda}_{k-1}^{(\alpha)}$ and $\underline{\mathrm{q}}_{\mathrm{q} . \mathrm{p} .2}=-\underline{\mathrm{e}}_{1}^{*}+\underline{\Lambda}_{k-1}^{(\beta)}$, where $\left\{\underline{\mathrm{e}}_{1}^{*}, \underline{\Lambda}_{i}^{(\alpha)}, \underline{\Lambda}_{i}^{(\beta)}, i=1 \ldots k-1\right\}$ is the basis of the dual decomposable lattice [13,14], with $\underline{\Lambda}_{i}^{(\alpha)}$ and $\underline{\Lambda}_{i}^{(\beta)}$ being the fundamental weights of the two $\mathrm{su}(k)$ algebras, and $\underline{\mathrm{e}}_{1}^{*}=\underline{\mathrm{Q}} / k[14]$. When we "fuse" the above mentioned quasiparticles, i.e., when we put all coordinates to the same point, the resulting object has a topological charge

$$
(\kappa M+1)\left(\underline{\mathrm{q}}_{\mathrm{q} \cdot \mathrm{p} \cdot 1}+\underline{\mathrm{q}}_{\mathrm{q} \cdot \mathrm{p} \cdot 2}\right)+\underline{\mathrm{q}}_{\mathrm{h}}=-\kappa\left(\underline{\Lambda}_{1}^{(\alpha)}+\underline{\Lambda}_{1}^{(\beta)}\right),
$$

which is just the sum of the above lattice charges. The right hand side of Eq. (19) is equivalent to $\underline{\Lambda}_{-\kappa}^{(\alpha)}+\underline{\Lambda}_{-\kappa}^{(\beta)}$ since for the $\operatorname{su}(k)$ weights one has $\square$ $a \underline{\Lambda}_{j} \simeq \underline{\Lambda}_{a j \bmod k}, a \in \mathbb{Z}$. Note that the combination with charge (19) carries zero electric charge (and, respectively, zero magnetic flux), however, it is not trivial since $\underline{\Lambda}_{-\kappa}$ does not belong to the $\operatorname{su}(2 \kappa)$ root lattice. Therefore, a neutral quasiparticle must be added in order to make the lattice charge (19) trivial; we note that the neutral composite (17), whose lattice charge is $\underline{q}_{\text {comp }}=$ $\underline{\Lambda}_{\kappa}^{(\alpha)}+\underline{\Lambda}_{\kappa}^{(\beta)}$, exactly compensates the topological charge excess in Eq. (19) provided that

$$
\underline{\Lambda}_{-\kappa}^{(\alpha)}+\underline{\Lambda}_{\kappa}^{(\alpha)}=\sum_{i=1}^{\kappa-1} i\left(\underline{\alpha}^{i}+\underline{\alpha}^{k-i}\right)+\kappa \underline{\alpha}^{\kappa} \simeq 0
$$

(and similarly for $\underline{\Lambda}^{(\beta)}$ ). In other words, the topological charge of the cluster

$$
1 \mathrm{comp}+1 \mathrm{~h}+(\kappa M+1)(\mathrm{q} \cdot \mathrm{p} \cdot 1+\mathrm{q} \cdot \mathrm{p} \cdot 2)
$$

6 recall that two $\mathrm{su}(k)$ weights are equivalent iff their difference belongs to the root lattice 
is trivial, i.e., $(\kappa M+1)\left(\underline{q}_{\text {q.p.1 }}+\underline{q}_{\text {q.p. } 2}\right)+\underline{q}_{h}+\underline{q}_{\text {comp }} \simeq 0$, so that $(20)$ is completely invisible in the adiabatic transport of any excitation around it, as shown on Fig. 1; this is equivalent to the statement (18). After taking the

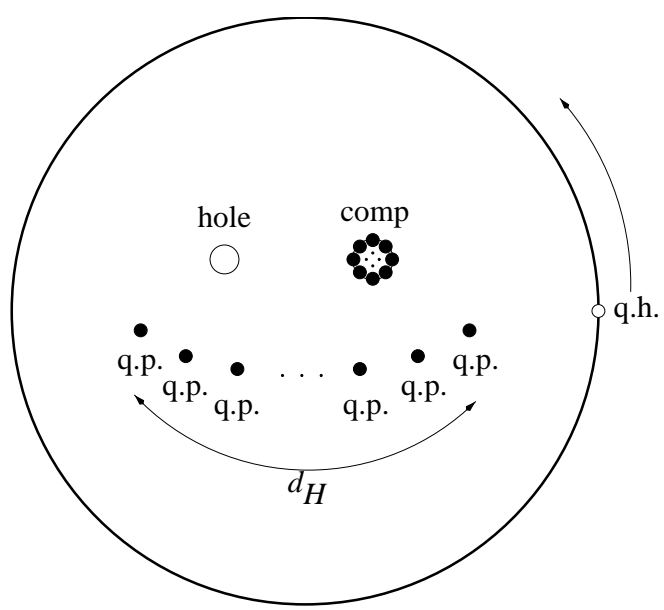

Fig. 1. The cluster consisting of one hole, $d_{H}=k M+2$ quasiparticles of both types (q.p.1 and q.p.2) and one neutral composite (comp) is topologically equivalent to the pair $(1 \mathrm{e}-1 \mathrm{~h})$ and is completely invisible in the adiabatic transport of quasiholes (q.h.) around it.

coset this picture does not change since the projection is supposed to preserve [14] the neutral component $\underline{\Lambda}_{1}^{(\alpha)}+\underline{\Lambda}_{1}^{(\beta)}$ of the physical hole and consequently preserves its $\kappa$-th fusion power. The difference is now that the quasiparticles of both types become indistinguishable and their statistics become non-abelian [14]. Nevertheless, the above adiabatic transport could be analyzed in a similar way taking into account the "non-abelian" fusion rules [14] leading to the same conclusion that the electron in the $k=2 \kappa$ case has a non-trivial neutral component given by (17).

Remark 2. The topological charge of the cluster (18), for the parafermion coset (6), has components in more than one super-selection sector due to the non-abelian fusion rules [17,4]. In that case, the charge of (18) must be projected onto the vacuum sector which is characterized by periodic/anti-periodic boundary conditions for the bosonic/fermionic currents.

One consequence of the chiral algebra extension is that the "extended " $\mathrm{QH}$ fluid is expected to be more stable than the original one. The point is that any extended algebra has less (or at least the same number) of IRs, as compared to the original one, since the IRs of the former must be constructed from those IRs of the latter which are in addition relatively local with respect to (17). According to the stability criterion S3 in [16], reducing the number of admissible IRs would further increase the stability of the $\mathrm{QH}$ fluid. Let us consider this in more detail. The operator product expansion of the field (16) with any coset IR's primary field $\Phi^{\mathrm{PF}}\left(\underline{\Lambda}_{\mu}+\underline{\Lambda}_{\rho}\right)$ is determined by the fusion rule 
[14] $\left(\underline{\Lambda}_{\kappa}+\underline{\Lambda}_{\kappa}\right) \times\left(\underline{\Lambda}_{\mu}+\underline{\Lambda}_{\rho}\right)=\left(\underline{\Lambda}_{\mu+\kappa}+\underline{\Lambda}_{\rho+\kappa}\right)$ (all indices are taken mod $\left.k\right)$ so that

$$
\Psi_{\kappa}^{(0)}(z) \Phi^{\mathrm{PF}}\left(\underline{\Lambda}_{\mu}+\underline{\Lambda}_{\rho}\right)(w) \sim(z-w)^{-\frac{\mu+\rho}{2}} \Phi^{\mathrm{PF}}\left(\underline{\Lambda}_{\mu+\kappa}+\underline{\Lambda}_{\rho+\kappa}\right)(w)
$$

where the power of $(z-w)$ is determined by matching the CFT dimensions (7) of both sides. Therefore, only those IR's primary fields of the original chiral algebra are relatively local with (16) which satisfy

$$
P=\mu+\rho=0 \bmod 2
$$

This "even $\mathbb{Z}_{k}$-charge restriction" reduces half of the IRs of the original algebra. It is not difficult to express the chiral partition functions $\tilde{\chi}_{l, \rho}(\tau, \zeta)$ corresponding to the extended algebra in terms of those (9) for the original one. First of all, the even-charge restriction (21) implies that the parameter $l$ in the original characters (9) must be even since $P\left(\underline{\Lambda}_{l-\rho+s}+\underline{\Lambda}_{\rho+s}\right)=2 s+l$ is supposed to be even. Second, since the only field in the extended algebra which does not belong to the original one is (16), naturally we have

$$
\begin{aligned}
\tilde{\chi}_{2 l, \rho}(\tau, \zeta) & \stackrel{\text { def }}{=} \chi_{2 l, \rho}(\tau, \zeta)+\chi_{2 l, \rho+\kappa}(\tau, \zeta)=\sum_{s=0}^{\kappa-1} K_{l+s(\kappa M+1)}(\tau, \kappa \zeta ; \kappa(\kappa M+1)) \times \\
& \times\left[\operatorname{ch}\left(\underline{\Lambda}_{l-\rho+s}+\underline{\Lambda}_{\rho+s}\right)+\operatorname{ch}\left(\underline{\Lambda}_{l-\rho+s+\kappa}+\underline{\Lambda}_{\rho+s+\kappa}\right)\right]
\end{aligned}
$$

where we have used the identity [14] $K_{2 l}(\tau, 2 \zeta ; 4 m)+K_{2 l+2 m}(\tau, 2 \zeta ; 4 m)=$ $K_{l}(\tau, \zeta ; m)$.

Remark 3. Only primary fields with half-integer or integer CFT dimensions can be used to extend the chiral algebra. Extending the algebra with $\Psi_{\kappa}^{(0)}$ (for $k=2 \kappa, \kappa$ integer) automatically extends the $\widehat{u(1)}$ algebra with $: \exp ( \pm i \sqrt{\kappa(\kappa M+1)} \phi(z))$ : because the corresponding quantum numbers $Q=$ $\pm \kappa, \Delta=\kappa(\kappa+1) / 2$ are found in the vacuum character $\tilde{\chi}_{0,0}(\tau, \zeta)$ for $s=0$ and the vacuum module is isomorphic to the chiral algebra. Therefore, the extension with the field (16) is the maximal one.

Next, we need to determine the possible values of $l$ and $\rho$ which give all the independent characters (22). We find

$$
\begin{aligned}
& \#\{2 l\}=\#\{0,2,4, \ldots,(k M+2)-2\}=\frac{k M+2}{2} \\
& \#\{\rho\}=\#\{\rho \mid 0 \leq \rho \leq \kappa-\rho\}=\left[\frac{\kappa}{2}\right]+1
\end{aligned}
$$


where $[x / 2]$ stands for the integer part of $x / 2$. The range of $2 l$ comes from the even-charge restriction (21). To understand how the range of $\rho$ is determined we first use the following symmetry of (22)

$$
\tilde{\chi}_{2 l, \rho+\kappa}(\tau, \zeta)=\tilde{\chi}_{2 l, \rho}(\tau, \zeta) \quad \Longrightarrow \quad 0 \leq \rho \leq \kappa-1
$$

to reduce the number of non-equivalent choices of $\rho$, and next consider only the $s=0$ term in (22) keeping in mind that the other terms are obtained by the action of the simple currents (expressed by the mod $\kappa$ sum in (22)) and represent the same IR of the full $\widehat{u(1)} \times \mathrm{PF}_{k}$ theory. In addition, since we are interested in the neutral degeneracy, we can fix an arbitrary value of charge, for example $2 l=0$, and consider the $s=0$ neutral character in $\widetilde{\chi_{0,0}}$

$$
\left[\operatorname{ch}\left(\underline{\Lambda}_{\rho}+\underline{\Lambda}_{k-\rho}\right)+\operatorname{ch}\left(\underline{\Lambda}_{\rho+\kappa}+\underline{\Lambda}_{\kappa-\rho}\right)\right]
$$

which could be labeled by the smallest of the $4 \underline{\Lambda}$ 's indices. We have $\rho<k-\rho$ and $\kappa-\rho \leq \kappa+\rho$, due to the range (25), so that the character (26) could be labeled by the smaller of the numbers $\rho, \kappa-\rho$. Thus we find the restriction for $\rho$ to be $0 \leq \rho \leq \kappa-\rho$ which gives $(24)$.

One important characteristics of the universality class of any $\mathrm{QH}$ fluid is the topological order [22]. In the $k=2 \kappa$ case this number is given by

$$
\mathrm{TO}(k=2 \kappa)=\#\{2 l\} \#\{\rho\}=(\kappa M+1)\left(\left[\frac{\kappa}{2}\right]+1\right)
$$

which is just the product of the two ranges (23) and (24). We stress that this number is smaller than the general- $k$ number (conjectured in [1] and derived in $[13,14])$

$$
\operatorname{TO}(k)=\frac{(k M+2)(k+1)}{2}
$$

computed for $k$ even, which is the main justification of the chiral algebra extension presented above since, according to the stability criterion S3 in [16], the less this number the more stable the fluid. (Note that the topological order for $k$ odd is still given by Eq. (28).)

Now, we can see the difference between the odd- $k$ and the even- $k$ cases. The coset primary field with the lowest non-zero charge and dimension for $k$ odd is labeled by $\underline{\Lambda}_{0}+\underline{\Lambda}_{1}$, while for $k$ even it is $\underline{\Lambda}_{0}+\underline{\Lambda}_{2}$; the reason is that $\underline{\Lambda}_{0}+\underline{\Lambda}_{1}$ does not satisfy the even-charge restriction (21), i.e., it is not relatively local with respect to (16). Therefore, the dimensions of the quasiholes for the $k$-even case would be given by a formula different from Eq. (15). Indeed, since the field 
$\Phi^{\mathrm{PF}}\left(\underline{\Lambda}_{0}+\underline{\Lambda}_{2}\right)$ is characterized by the $s=0$ term in $\tilde{\chi}_{2,0}(\tau, \zeta)$ the elementary charge and CFT-dimension is (according to Eq. (14) for $M=1$, and Eq. (11) and (12) for $s=0, l=2, \rho=0$ )

$$
Q_{k=2 \kappa}^{\text {q.h. }}=\frac{2}{k+2}, \quad \Delta_{k=2 \kappa}^{\text {q.h. }}=\frac{1}{k+2} .
$$

For clarity, the quasiholes' charges and dimensions for the first 10 plateaux in the parafermionic hierarchies (1) and (2) are given in Table 1.

We stress here that the quantum numbers $P$ in Eq. (8), of the parafermionic currents $\Phi^{\mathrm{PF}}\left(\underline{\Lambda}_{\mu}+\underline{\Lambda}_{\mu}\right)$ in the $k=2 \kappa$ case generate only an even-charge subgroup $\mathbb{Z}_{\kappa} \subset \mathbb{Z}_{2 \kappa}$. In this case, however, one can redefine the parafermion charge (8) as follows

$$
P^{\prime}\left(\underline{\Lambda}_{\mu}+\underline{\Lambda}_{\rho}\right) \stackrel{\text { def }}{=} \frac{P\left(\underline{\Lambda}_{\mu}+\underline{\Lambda}_{\rho}\right)}{2} \bmod k
$$

for $\mu+\rho=0 \bmod 2$, in agreement with Eq. (21). The new charge $P^{\prime}$ belongs to $\mathbb{Z}_{2 \kappa}$ and is preserved under the fusion rules [14]. In addition, for all IRs satisfying Eq. (21), one can define a chiral fermion parity according to $\gamma_{F}=$ $P^{\prime} \bmod 2$.

In particular, the composite particles (17) have a $\mathbb{Z}_{k}$ charge $P\left(\underline{\Lambda}_{\kappa}+\underline{\Lambda}_{\kappa}\right)=2 \kappa=$ 0 mod $k$, while their modified parafermion charge (30) is $P^{\prime}\left(\underline{\Lambda}_{\kappa}+\underline{\Lambda}_{\kappa}\right)=\kappa$ and thus $\gamma_{F}=\kappa \bmod 2$, in agreement with the discussion after Eq. (17).

\section{Relations between charge, spin and statistics of QH quasiparti- cles}

Despite the fact that the electrons in a QH fluid are non-relativistic their lowenergy collective excitations (quasiparticles and quasiholes) are believed [21] to satisfy a generalized spin-statistics relation [30]

$$
2 \Delta_{\text {q.h. }}=\frac{\theta_{\text {q.h. }}}{\pi}=S_{\text {q.h. }}+S_{\text {q.p. }}
$$

(in general this must be a $\bmod \mathbb{Z}$ relation, however, for the elementary quasiholes (14) and quasiparticles it is exact [30]), where the CFT dimension $\Delta_{\text {q.h. }}=\Delta_{\text {q.p. }}$ is actually the average spin of the pair (quasiparticle-quasihole) and $\theta_{\text {q.h. }}$ is the statistical angle. Eq. (31) explains why the CFT dimensions can be identified with the quantum statistics of the quasiparticles. 
On the other hand, there is a very interesting relation between the charge and the statistics of the QH quasiparticles corresponding to the Read-Rezayi states, namely, the electric charges (11) and CFT dimensions (12) are related (we set $M=1$ for simplicity) by a generalized charge-statistics relation

$$
2 \Delta=Q+\frac{2 n}{d_{H}}
$$

where $d_{H}=k+2$ is the denominator of the filling factor and

$$
2 n=d_{H}[\mu(\mu+1)+2 b \mu]+2 b\left(\rho^{\prime}-\mu\right)+k b(b-1)
$$

is an even integer. Given the parameters $l, \rho$ and $s$ of the excitation (10), the new parameters $\mu, \rho^{\prime} \in \mathbb{Z}_{k}$, and $a, b, c \in \mathbb{Z}$ entering Eq. (33) are uniquely determined from

$$
\begin{aligned}
& \min (l-\rho+s, \rho+s)=\mu+a k, \quad b=s-\mu+a+c \\
& \max (l-\rho+s, \rho+s)=\rho^{\prime}+c k .
\end{aligned}
$$

Note that the elementary quasiholes $(l=1, \rho=0, s=0$ for $k$ odd and $l=2$, $\rho=0, s=0$ for $k$ even) satisfy Eq. (32) with $n=0$, i.e.,

$$
2 \Delta_{\text {q.h. }}=Q_{\text {q.h. }} .
$$

The elementary quasiparticles $(l=-1, \rho=0, s=0$ for $k$ odd and $l=-2$, $\rho=0, s=0$ for $k$ even) satisfy Eq. (32) with $n=1$, and $n=2$ respectively, however, in both cases one has

$$
2 \Delta_{\text {q.p. }}=\left|Q_{\text {q.p. }}\right|
$$

Both Eqs. (34) and (35) together with the spin-statistics relation (31) explicitly relate the fractional charge of the (elementary) QH quasiparticles to their fractional statistics [21]. This looks natural because spin in $2 \mathrm{D}$ is an additive quantum number (like the electric charge), though this additivity, which in CFT language is called "matching CFT dimensions", is masked in OPEs by the powers of the distance (in other words, spin can partially transform into relative orbital momentum when building a composite particle). Nevertheless, for "indecomposable" primary fields, such as the quasihole, which cannot be generated in the right hand side of an OPE of other fields with smaller CFT dimensions, this additivity amounts to a linear relation between charge and spin. We stress that the quantum statistics of a quasiparticle is usually computed by Berry phase technique $[4,21]$, however, this computation explicitly 
uses the quasiparticle wave functions that are in general not known. The advantage of the generalized charge-statistics relation (34) is that it determines the quasihole's CFT dimension (respectively, statistics) in terms of the minimal electric charge, which is fixed by the filling factor. This gives another viewpoint on the relation between the precisely quantized (fractional) Hall conductivity and the sharp quantization of the (fractional) electric charge.

It is important to note that general multi-electron clusters that are bosons/fermions for even/odd electric charge satisfy Eq. (32) with $n=m d_{H}(m \in \mathbb{Z})$ thus reproducing the standard charge-statistics relation (see e.g. Eq. (31) in [16]).

Finally, we expect that the generalized charge-statistics relations (32), (34) and (35) go beyond the scope of the Read-Rezayi states in the second Landau level. We believe that such relations are fundamental characteristics of the $\mathrm{QH}$ fluids [31] and we expect them to hold also in the principle series $\nu=n /(2 n+1)$ of QH filling fractions.

\section{Energy gaps for the parafermion QH fluids}

In this section we compute the energy gaps for the parafermionic Hall states in the second Landau level. To this end, we use Eq. (5) with $\Delta_{\text {q.h. }}$ derived from the effective field theory at the edge. Before going into the details of the calculation we have to make several remarks. First of all, the number $(2 n+1)^{-1}$, which appears in the gap formula Eq. (3) in [25], is generically the charge of the quasihole. In our case, however, this charge turns out to be twice the CFT dimension of the quasihole operator (see Eqs. (34) and (35)) which justifies the ansatz (5). It is worth-recalling the "conventional wisdom" [25] that (i) the non-zero thickness in the transversal direction and Landau level mixing simply renormalize $\alpha$ in (5) (ii) the Landau level broadening due to the residual disorder simply reduces the gap by $\Gamma$ and (iii) $\alpha$ and $\Gamma$ are approximately independent of $k$ (resp. of $\nu_{k}$ ). Second, we recall that usually the conformal dimensions are related to the energies of the edge excitations, whose effective chiral Hamiltonian is [28] $v\left(L_{0}-c / 24\right) / R$, where $L_{0}$ is the zero mode of the Virasoro stress tensor, $v$ is the edge states velocity and $R$ the radius of the edge circle, i.e., all excitations become gapless in the thermodynamic limit $R \rightarrow \infty$. However, since this CFT describes the edge of an incompressible QH fluid, where the bulk charge-density waves are suppressed by an energy gap, this means that any localized charged excitation in the bulk is completely transmitted to the edge and is seen there as an edge excitation carrying all quantum numbers (such as topological charges and quantum statistics) of its bulk counterpart. The point is that the ideal gap is proportional to some universal quantity, such as the quasiparticle's electric charge, which can be computed at the edge. 
We speculate that Eq. (5) admits an interpretation of the energy gap as the centrifugal barrier for creating the minimal spin dictated by the statistics of quasiparticles, which, on the other hand, is determined by the filling factor. Indeed, if we assume that the quasiparticle energy gap is proportional to its spin, then the average gap $\epsilon$ of the pair (q.p.-q.h.) is proportional to the average spin, which, according to the spin-statistics relation (31), is exactly equal to the quantum statistics [30], respectively, to the CFT dimension of the quasihole

$$
\frac{\epsilon_{\text {q.p. }}+\epsilon_{\text {q.h. }}}{2} \sim \frac{S_{\text {q.p. }}+S_{\text {q.h. }}}{2}=\frac{\theta_{\text {q.h. }}}{2 \pi}=\Delta_{\text {q.h. }}
$$

In realistic cases, one has to take into account the non-universal effects (see (i), (ii) and (iii) above) as in Eq. (5). Next, the charge-statistics relations (34) and (35) relate the (ideal) energy gap to the electric charge of the elementary quasiparticle, which is equal to the denominator of the filling factor, thus reproducing Eq. (3) in [25].

Now, let us go back to Eq. (5). The two constants $\alpha$ and $\Gamma$ could be determined by fitting the measured energy gaps for the two most stable plateaux $k=2$, $\widetilde{\Delta}_{2}=0.110 \mathrm{~K}$ and $k=4, \widetilde{\Delta}_{4}=0.055 \mathrm{~K}$. We find that

$$
\alpha=0.0063, \quad \Gamma=0.045 \mathrm{~K}
$$

which allows us to compute the other activation gaps in the hierarchies (1) and (2) for the sample of [15]. Note that $\alpha$ and $\Gamma$ (depending on the particle density and layer thicknes) are not universal and must be re-fitted for any other sample.

The more important data about the plateaux (1) in the sample of [15] is summarized in Table 1 . In Table 2 we give the similar data for the $\mathrm{PH}$ conjugate plateaux (2). In both tables $M=1$, the average electron density $n_{e} \simeq 2.2 \times 10^{11}$ $\mathrm{cm}^{-2}$ is taken from [15], the magnetic field, $B_{k}$ for the hierarchy(1) and $B_{k}^{\prime}$ for the hierarchy(2), is measured in T. The quasiholes' charges $Q_{k}^{\text {q.h. }}$ are measured in units in which the electron charge is -1 . The CFT dimensions of the quasiholes are $\Delta_{k}^{\text {q.h. }}, \mathrm{TO}(k)$ is the topological order and the measurable activation energy gaps $\widetilde{\Delta}_{k}$ and ${\widetilde{\Delta^{\prime}}}_{k}$ are given in ${ }^{\circ} \mathrm{K}$. The gaps are given to 2 significant figures because the gaps for $k=2$ and $k=4$ were measured with this precision and in order to be able to compare these predictions with the experiment. The values of the quasiholes' charges $Q_{k}^{\text {q.h. }}$, CFT dimensions $\Delta_{k}^{\text {q.h. }}$ and topological order $\mathrm{TO}(k)$ in Table 2 are the same like those corresponding to the same $k$ in Table 1.

The $k=2$ plateau is common for both Tables since it is self-conjugate. The $8 / 3$ plateau appears as $k=4$ in Table 1 and as $k=1$ in Table 2 , while the 
Table 1

Quasiholes' charges and CFT dimensions, topological orders and the measurable activation energy gap for the parafermionic states at filling factors (1) for the sample of [15].

\begin{tabular}{c||cccccccccc}
\hline$k$ & 1 & 2 & 3 & 4 & 5 & 6 & 7 & 8 & 9 & 10 \\
\hline \hline$\nu_{k}$ & $7 / 3$ & $5 / 2$ & $13 / 5$ & $8 / 3$ & $19 / 7$ & $11 / 4$ & $25 / 9$ & $14 / 5$ & $31 / 11$ & $17 / 6$ \\
\hline$B_{k}$ & 3.92 & 3.66 & 3.52 & 3.43 & 3.37 & 3.32 & 3.29 & 3.26 & 3.24 & 3.23 \\
\hline$Q_{k}^{\text {q.h. }}$ & $1 / 3$ & $1 / 2$ & $1 / 5$ & $1 / 3$ & $1 / 7$ & $1 / 4$ & $1 / 9$ & $1 / 5$ & $1 / 11$ & $1 / 6$ \\
\hline$\Delta_{k}^{\text {q.h. }}$ & $1 / 6$ & $1 / 4$ & $1 / 10$ & $1 / 6$ & $1 / 14$ & $1 / 8$ & $1 / 18$ & $1 / 10$ & $1 / 22$ & $1 / 12$ \\
\hline $\mathrm{TO}(k)$ & 3 & 2 & 10 & 6 & 21 & 8 & 36 & 15 & 55 & 18 \\
\hline$\widetilde{\Delta}_{k}$ & 0.062 & 0.110 & 0.015 & 0.055 & -0.003 & 0.029 & -0.012 & 0.013 & -0.019 & 0.003 \\
\hline
\end{tabular}

Table 2

The measurable activation energy gap for the $\mathrm{PH}$ conjugate plateaux of the parafermionic states at filling factors (2) for the sample of [15].

\begin{tabular}{c||cccccccccc}
\hline$k$ & 1 & 2 & 3 & 4 & 5 & 6 & 7 & 8 & 9 & 10 \\
\hline \hline$\nu_{k}^{\prime}$ & $8 / 3$ & $5 / 2$ & $12 / 5$ & $7 / 3$ & $16 / 7$ & $9 / 4$ & $20 / 9$ & $11 / 5$ & $24 / 11$ & $13 / 6$ \\
\hline$B_{k}^{\prime}$ & 3.43 & 3.66 & 3.81 & 3.92 & 4.00 & 4.06 & 4.11 & 4.15 & 4.19 & 4.22 \\
\hline$\widetilde{\Delta^{\prime}}{ }_{k}$ & 0.055 & 0.110 & 0.018 & 0.062 & 0.001 & 0.037 & -0.09 & 0.021 & -0.015 & 0.010 \\
\hline
\end{tabular}

plateau $7 / 3$ appears in reverse order. Notice that the $k=1$ states are actually a Laughlin states, as mentioned in [1]. This is directly seen from the $k$-body interaction [1], as well as from the coset (6), which gives trivial neutral part and the remaining $\mathrm{CFT}$ is $\widehat{u(1)}$ with compactification radius 3 . We see that, in general, the gaps for the PH-conjugate states (2) are slightly bigger than those for (1) because the magnetic length in the former is smaller than that in the latter. However, the experimental gap $(0.10 \mathrm{~K})$ for $\nu_{4}^{\prime}=7 / 3$ is much bigger [15] than the gap $(0.055 \mathrm{~K})$ of its conjugate, the $\nu_{4}=8 / 3$ one, which cannot be explained by the smaller magnetic length only. This can be explained by the fact that the Laughlin states for $k=1$ have bigger gaps than the $k=4$ parafermion states, according to the stability criterion S3 in [16], because the topological order of the former is 3 while that for the latter is 6 (after the extension). Nevertheless, this makes no difference in Eq. (5) since the electric charges of the quasiholes of both $k=1$ and $k=4$ states are equal.

We stress that it is in first approximation that $\alpha$ and $\Gamma$ in Eq. (5) are independent of the filling factor, so that one cannot expect a perfect overlap with the experiment. If we accept, comparing to the result in [25], that the accuracy is not better than $30 \%$ then the gap for the plateau at $\nu=13 / 5$ should be $15 \pm 5 \mathrm{mK}$, while that for $\nu=7 / 3$ is $62 \pm 20 \mathrm{mK}$. Nevertheless, this approximation seems to be sufficient to determine the structure of plateaux 
in the parafermionic hierarchy. Note that without the extension of the chiral algebra for $k$ even, the gap for $\nu=13 / 5$, computed by our gap ansatz (5), would be $\simeq 77 \pm 25 \mathrm{mK}$, which obviously contradicts the experimental data [15], which suggests that this value is $\sim 10 \mathrm{mK}$.

Taking into account that the electron temperature $T_{e}$ at which $\nu_{2}=5 / 2$ is already quantized is [15] $T_{e} \simeq 8 \mathrm{mK}$, we expect that the $\nu_{3}=13 / 5$ plateau quantization could be observed at $T_{e} \simeq 1 \mathrm{mK}$ in the same sample. In addition, the $k=8$ state $\left(\nu_{8}=14 / 5\right)$ should be approximately as stable as the $k=3$ one $\left(\nu_{3}=13 / 5\right)$ (apart from the difference in the magnetic length) and is also expected to be observed at that temperature.

We also conjecture that the $R_{x x}$ minimum around $B \simeq 3.35 \mathrm{~T}$ corresponds to $\nu_{6}=11 / 4$ rather than to $\nu_{5}=19 / 7$ and more precise measurements down to electron temperatures around $1.5 \mathrm{mK}$ would be necessary in order to confirm this. In the same way, the $R_{x x}$ minimum around $B \simeq 4.05 \mathrm{~T}$ corresponds $\nu_{6}^{\prime}=9 / 4$ rather than to $\nu_{5}^{\prime}=16 / 7$. The plateaux with $k>5$ as well as the

$\nu=19 / 7$ one $(k=5$ in (1)) are not expected to be observed in the sample of [15], even at zero temperature, since the gap reduction $\Gamma$ in Eq. (5) is bigger than the (renormalized) ideal gap. The plateaux with even $k=10(\nu=17 / 6$ and $\left.\nu^{\prime}=13 / 6\right)$ are neither expected to be seen, despite the fact that their gaps are big enough, because of the overlaps with the wide integer Hall plateaux at $\nu=3$ and 2 respectively. The last plateau in the series (1) observable in the sample of [15] is expected to be $14 / 5(k=8)$ for the hierarchy (1) and $11 / 5$ $(k=8)$ for $(2)$.

An important message to the experiment is to measure the activation gaps for a high-mobility sample at temperatures down to $1 \mathrm{mK}$, which seem to be accessible [32]. We disagree with the conclusion in [15] that "the gaps for $\nu=5 / 2,8 / 3$ and $7 / 3$ are approximately equal and on the order of $2 \mathrm{~K}$ ". Therefore, it would be interesting to check experimentally our predictions for the gaps at $\nu=7 / 3,13 / 5,12 / 5,16 / 7,11 / 4,9 / 4,14 / 5$ and $11 / 5$.

\section{Summary and discussion}

We have demonstrated that the non-monotonic plateaux structure of the parafermionic QH states in the second Landau level can be understood in terms of the effective CFT for the edge states. The main idea is to find the maximal chiral algebra extension since this increases the stability (respectively, the energy gap) of the QH fluid, according to the stability criterion S3 in [16]. However, only bosons and fermions can be used for that purpose and the key observation is that for even $k$ there are neutral composite bosons/fermions among the excitations of the parafermion $\mathrm{QH}$ states, which can extend the 
algebra. This reduces the topological order and increases the minimal electric charge, respectively, the activation energy gap.

The detailed analysis of the spectrum of the parafermion edge excitations shows that, in addition to the generalized spin-statistics relation (31), there is an exact charge-statistics relation for the elementary quasiparticles (35) and quasiholes (34). These two relations together with the assumption that the pure gap is proportional to the minimal electric charge, like in the principle series of filling factor [25], allow us to compute the measurable activation gaps for all plateaux in the hierarchies (1) and (2) for the sample of [15]. In simple words, assuming that the minimal electric charge for $k$ even is twice bigger, gives an explanation of the non-monotonic structure of the parafermion plateaux.

We stress that the gap reduction $\Gamma$ in Eq. (36), that we computed for the sample of [15], appears to be 2 orders of magnitude less than the value estimated in [15]. Although it seems plausible that this non-universal quantity is smaller for the extremely high quality sample of [15] (since it depends more on the non-zero thickness than on the residual disorder [25]) we have to make clear that if the realistic gap reduction is bigger, then our argument becomes qualitative. In other words, even if Eq. (5) is not a good approximation, the fact that the extended fluids for $k$ even are more stable than the original ones, still may explain the non-monotonic structure of the observed plateaux. In order to check our predictions new activation experiments, down to electron temperature of $1.5 \mathrm{mK}$, are necessary.

For the $\nu=5 / 2$ state $(k=2)$ our stability analysis implies that the elementary quasiholes have electric charge $1 / 2$ and obey abelian fractional statistics $\theta=\pi / 2$. Then the ordinary electron splits into two quasiparticles plus one Majorana fermion at the edge $\square$. The ground state wave function is still given by the Pfaffian $[4,5,8,7]$, however, the structure of the quasihole excitations is different [31]. Thus, the extended rational CFT discussed in Sect. 2 defines a new universality class for the $\nu=5 / 2 \mathrm{QH}$ state, which is clearly distinguished from the MR one. On the other hand, recent numerical calculations [33] have shown that the numerical ground state for the pseudopotential Hamiltonian has an excellent overlap with the MR state, which means that most likely at $T=0$ the true $\nu=5 / 2 \mathrm{QH}$ state belongs to the MR universality class. However, one thing characterizing the MR state is that the $\mathbb{Z}_{2}$ symmetry corresponding to the conservation of the chiral fermion parity is spontaneously broken [31]. As temperature increases, this symmetry may be restored driving the system into the abelian phase described above (in which chiral fermion

$\overline{7}$ This is not surprising since even in the $K$-matrix theory the quasiparticle topological charge is not collinear with that of the electron (see Eq. (18)) so that a neutral component is always present. 
parity is conserved). Thus, one may expect a phase transition [31] between the two phases, which could be easily detected since the minimal electric charge changes from $1 / 4$ to $1 / 2$. This may possibly explain the kink at $15 \mathrm{mK}$ observed in the activation experiment [15].

Thus, we conclude that precise quasiparticle charge-measuring experiments, such as shot-noise, especially for temperature in the range $10 \div 30 \mathrm{mK}$ and a high-mobility sample similar to that of [15], as well as new activation experiments down to temperature $1 \mathrm{mK}$, could be really decisive for revealing the nature of the enigmatic $\nu=5 / 2$ state.

\section{Acknowledgements}

I would like to thank Andrea Cappelli, Ivan Todorov and Jürg Fröhlich for fruitful discussions, Wei Pan for kindly offering details from the experiment and the Max-Planck-Institute für Physik Komplexer Systeme in Dresden for hospitality and financial support. This work was supported by DFG through Schwerpunktprogramm "Quanten-Hall-Systeme" under the program "Konforme Feldtheorie der Quanten-Hall-Plateau-Übergänge".

\section{References}

[1] N. Read and E. Rezayi Phys. Rev. B59 (1998) 8084.

[2] R. Willett, J. P. Eisenstein, H. L. Störmer, D. C. Tsui, A. C. Gossard, and J. H. English, "Observation of an even-denominator quantum number in the fractional quantum Hall effect," Phys. Rev. Lett. 59 (1987) 1776.

[3] J. Eisenstein, R. Willett, H. L. Störmer, D. C. Tsui, A. C. Gossard, and J. H. English, "Collapse of the even-denominator fractional quantum Hall effect in tilted fields," Phys. Rev. Lett. 61 (1988) 997.

[4] G. Moore and N. Read Nucl. Phys. B360 (1991) 362.

[5] M. Milovanović and N. Read, "Edge excitations of paired fractional quantum Hall states," Phys. Rev. B 54 (1996) 13559, cond-mat/9612147.

[6] R. Morf, "Transition from quantum Hall to compressible states in the second landau level: new light on the $\nu=5 / 2$ enigma," Phys. Rev. Lett. 80 (1998) 1505, cond-mat/9809024.

[7] A. Cappelli, L. S. Georgiev, and I. T. Todorov, "A unified conformal field theory description of paired quantum Hall states," Commun. Math. Phys. 205 (1999) 657, hep-th/9810105. 
[8] N. Read, "Paired fractional quantum Hall states and the $\nu=5 / 2$ puzzle," Physica B298 (2001) 121, cond-mat/0011338.

[9] A. Zamolodchikov and V. Fateev ZhETF 89 (1985) 380.

[10] V. Fateev and A. Zamolodchikov Nucl. Phys. B280 (1987) 644.

[11] V. Fateev and S. Lykyanov Int. J. Mod. Phys. A3 (1988) 507.

[12] V. Gurarie and E. Rezayi, "Parafermion statistics and quasihole excitations for the generalizations of the paired quantum Hall states," Phys. Rev. B61 (2000) 5473, cond-mat/9812288.

[13] A. Cappelli, L. Georgiev, and I. Todorov, "Coset construction of parafermionic Hall states," Proc. of Supersymmetries and Quantum Symmetries SQS'99, July 1999, Dubna JINR (2000) 235, ESI 828.

[14] A. Cappelli, L. Georgiev, and I. Todorov, "Parafermion Hall states from coset projections of abelian conformal theories," Nucl. Phys. B599 (2001) 499, hep-th/0009229.

[15] W. Pan, J.-S. Xia, V. Shvarts, D. E. Adams, H. L. Störmer, D. C. Tsui, L. N. Pfeiffer, K. W. Baldwin, and K. W. West, "Exact quantization of the even-denominator fractional quantum Hall state at $\nu=5 / 2$ landau level filling factor," Phys. Rev. Lett. 83 (1999) 3530.

[16] J. Fröhlich, B. Pedrini, C. Schweigert, and J. Walcher, "Universality in quantum Hall systems: Coset construction of incompressible states," J. Stat. Phys. 103 (2001) 527, cond-mat/0002330.

[17] P. Di Francesco, P. Mathieu, and D. Sénéchal, Conformal Field Theory. Springer-Verlag, New York, 1997.

[18] X.-G. Wen Int. J. Mod. Phys. B4 (1990) 239.

[19] J. Fröhlich and T. Kerler Nucl. Phys. B354 (1991) 369.

[20] J. Fröhlich, U. M. Studer, and E. Thiran J. Stat. Phys. 86 (1997) 821, cond-mat/9503113.

[21] F. Wilczek, Fractional statisitcs and anyon superconductivity. World Scientific, Singapore, 1990.

[22] X.-G. Wen Adv. Phys. 44 (1995) 405.

[23] A. Cappelli, C. A. Trugenberger, and G. R. Zemba, "Stable hierarchical quantum Hall fluids as $W_{1+\infty}$ minimal models," Nucl. Phys. B448 (1995) 470, hep-th/9502021.

[24] J. Fuchs, B. Schellekens, and C. Schweigert Nucl. Phys. B461 (1996) 371, hep-th/9509105.

[25] K. Park and J. Jain Phys. Rev. Lett. 81 (1998) 4200.

[26] P. Goddard, A. Kent, and D. Olive Commun. Math. Phys. 103 (1986) 105. 
[27] D. Gepner and Z. Qiu Nucl. Phys. B285 (1987) 423.

[28] A. Cappelli and G. R. Zemba, "Modular invariant partition functions in the quantum Hall effect," Nucl. Phys. B490 (1997) 595, hep-th/9605127.

[29] A. Berkovich and B. M. McCoy, "The universal chiral partition function for exclusion statistics," Stat. Phys. on the Eve of the 21st Century, Series in Adv. Stat. Mechanics 14 (1999) 240-256, hep-th/9808013.

[30] J. Leinaas, "Spin and statistics of quantum Hall quasi-particles," in Confluence of Cosmology, Massive Neutrinos, Elementary Particles, and Gravitation, Ed. Kursunoglu et al., Kluwer Academic/Plenum Publisher, New York (1999) cond-mat/9903329.

[31] L. Georgiev, "The $\nu=5 / 2$ quantum Hall state revisited," (2001) hep-th/0108173.

[32] F. Ertl (in preparation).

[33] E. Rezayi and F. Haldane, "Incompressible paired Hall state, strip order and composite fermion liquid phase in half-filled landau level," Phys. Rev. Lett. 84 (2000) 4865, cond-mat/9906137. 